\title{
Fertility Preservation in Premature Ovarian Insufficiency (POI) Secondary to FSH Receptor Gene (FSHR) Mutation: Is There a New Hope?
}

\author{
Pierre-Antoine Pradervand1,2, Roland Antaki ${ }^{1,2}$, Simon Phillips ${ }^{1,2}$, C. Sylvestre ${ }^{1,3}$, Zaki El Haffaf', \\ Ariane Godbout ${ }^{1,2}$, Isaac Jacques Kadoch ${ }^{1,2}$, Pierre Lehmann ${ }^{1,4}$ \\ ${ }^{1}$ OVO Fertility, OVO Clinic, Montreal, Canada \\ ${ }^{2}$ Assisted Procreation Clinic, Centre Hospitalier de l'Université de Montréal (CHUM), Montreal, Canada \\ ${ }^{3}$ Division of Reproductive Endocrinology and Infertility, Centre Hospitalier Universitaire (CHU) Ste-Justine, \\ Montreal, Canada \\ ${ }^{4}$ Department of Obstetrics and Gynecology, Hôpital Sacré Coeur de Montréal, Montreal, Canada \\ Email: papradervand@lafondation.ch
}

How to cite this paper: Pradervand, P.-A., Antaki, R., Phillips, S., Sylvestre, C., El Haffaf, Z., Godbout, A. Kadoch, I.J. and Lehmann, P. (2017) Fertility Preservation in Premature Ovarian Insufficiency (POI) Secondary to FSH Receptor Gene (FSHR) Mutation: Is There a New Hope? Case Reports in Clinical Medicine, 6, 274-280. https://doi.org/10.4236/crcm.2017.610030

Received: August 17, 2017

Accepted: October 20, 2017

Published: October 23, 2017

Copyright (c) 2017 by authors and Scientific Research Publishing Inc. This work is licensed under the Creative Commons Attribution International License (CC BY 4.0).

http://creativecommons.org/licenses/by/4.0/

\begin{abstract}
A 19 years old patient with primary amenorrhea was referred to our center. Based on discrepancy between high follicle stimulating hormone (FSH) level and normal ovarian reserve parameters, follicle stimulating hormone receptor (FSHR) mutation was screened. The patient was homozygous in exon 6 of the FSHR gene for the new variant c.479T $>\mathrm{C}$ and predicted to result in an aminoacid substitution p.Ile160Thr. One year later, her anti-müllerian hormone (AMH) level inexplicably decreased. Oocyte vitrification was thus offered for fertility preservation. After 17 days of recombinant follicle stimulating hormone (recFSH) (900 IU daily), no follicular growth was seen and estradiol levels remained low. In vitro maturation (IVM) was then suggested. Ten oocytes were successfully vitrified.
\end{abstract}

\section{Keywords}

FSH Receptor Mutation, Fertility Preservation, in Vitro Maturation, AMH, Primary Ovarian Insufficiency

\section{Introduction}

Primary ovarian insufficiency patients represent a major challenge for obstetricians and gynecologist in terms of fertility management. Even if it is rare, when POI is combined with a FSHR mutation, helping patient in her family planning looks almost impossible. If POI is most often of unknown etiology, the incidence 
of family-related cases can achieve a percentage from $4 \%$ to $31 \%$, suggesting a genetic etiology in a large proportion of patients [1] [2].

In 1995, Aittomäki et al. observed a particular Finnish population presenting with elevated gonadotropin levels and primary amenorrhea but a normal karyotype, ending in a POI [3]. Associated with streaks ovaries, this condition is called ovarian dysgenesis (ODG). Actually, Aittomäki et al. linked for the first time the ODG to a special FSH receptor (FSHR) mutation. They reported that a C566T transition in exon 7 of FSHR, predicting an Ala to Val substitution at residue 189 in the extracellular ligand-binding domain, was congruent with the ovarian dysgenesis observed in the Finnish population for homozygous women. More precisely, the locus segregating the disease can be mapped on chromosome number $2 \mathrm{p}$, which corresponds to the locus for both FSHR and luteinizing hormone receptor (LHR) [4]. Other research in the western countries couldn't find the same mutation in women with the same phenotype, suggesting that the finish case was very peculiar [5]. Beau et al. yet, identified another FSHR mutation in a woman with a secondary amenorrhea, elevated gonadotropins, a subnormal estrogenic activity and normal ovaries, a different phenotype than the ODG [6]. Since then, a few FSHR mutation studies were reported in the literature, and most of them highlight the link between FSHR mutation or polymorphism and an ovarian dysfunction that could present under different forms [4] [7].

To date, when such a mutation was suspected, ovarian biopsy was often suggested as the diagnostic tool and virtually no fertility treatment could be offered, but we now seem to have more to offer to these patients.

\section{Case Presentation}

A 19 years old patient with primary amenorrhea was referred to our center. She measured $166 \mathrm{~cm}$ and weighed $57 \mathrm{kgs}$ (body mass index 21.8). She had moderately developed secondary sex characteristics interesting particularly breasts, and diminished sized ovaries and uterus. She had normal axillary and pelvic hair, and had no morphotypic abnormalities. At this time, the antral follicular count $(\mathrm{AFC})$ was $16(8+8)$ and the anti-müllerian hormone $(\mathrm{AMH})$ was 1.59 $\mathrm{ng} / \mathrm{ml}$.

Based on a discrepancy between high early follicular phase FSH level (72.5 IU/L) (ref. range 3.1 - 7.9 IU/L) and normal other ovarian reserve parameters, such as AMH levels and AFC, a FSHR mutation was suspected and searched for. A genetic screening was also performed for Fragile X Mental Retardation 1 gene (FMR1) premutation and for her karyotype. We furthermore completed an investigation of the classic auto-immune and endocrinologic causes of POF.

We found no FMR1 mutation or premutation and the karyotype was 46XX. The biological investigations were all normal. However, the patient was homozygous in exon 6 of the FSHR gene for a variant defined as c.479T > C and predicted to result in the amino acid substitution p.Ile160Thr. Her parents were non-consanguineous French Canadians and were carrying the same mutation. 
They were healthy and the mother had no complications during her pregnancy or delivery.

One year later, her AMH level decreased from 1.59 to $0.62 \mathrm{ng} / \mathrm{ml}$ and her AFC from 16 to 9 for unexplained reason, so that a fertility preservation using oocyte vitrification was offered. Being aware of the FSHr mutation, we started with a stimulated cycle nonetheless. After 17 days of 900 IU daily doses of recombinant FSH (recFSH), no follicular growth was seen and estradiol levels remained low. Therefore an in vitro maturation (IVM) was done, with the use of subcutaneous hCG (10.000 IU) 34 hours before the ovarian function.

Three Further IVM cycles were carried out to maximize the number of vitrified mature oocytes. She firstly underwent five days of stimulation with daily doses of recFSH (300 IU) and recombinant luteinizing hormone (recLH) (300 IU) before the ovulation induction.

In vitro maturation was carried out using the following technique: follicular fluid was aspirated from small follicles (between $3 \mathrm{~mm}$ and $8 \mathrm{~mm}$ ), the fluid was assessed by the embryologist using a $22 \mu \mathrm{m}$ filter to maximise oocyte retention. Once the oocyte collection was completed, immature oocytes were washed using Global Total (IVFonline, Canada) and then transferred into the prepared IVM media. IVM media was made up on the morning of the oocyte collection, one 75 IU ampoule of Repronex (Ferring, Canada) was diluted and using to produce a $0.75 \mathrm{IU}$ solution for the oocyte culture.

Immature oocytes were cultured for 25 hours in the maturation media and then assessed for cumulus expansion and extrusion of the first polar body; this was facilitated by sliding the oocyte. Sliding refers to visualizing the oocyte in a very thin layer of media created by tipping the dish gently to the side leaving the oocyte to flatten in the dish. Oocytes judged to be mature were denuded of their cumulus cells using recombinant hyaluronidase (Cumulase, Origio) and subjected to ICSI. Immature oocytes were further cultured for an additional 24 hours and assessed for maturation at which time they were denuded and ICSI was performed. Following ICSI, oocytes were transferred into our standard culture media system (Global, IVFonline, Canada).

In this 19 years old patient with a hypergonadotropic hypogonadism and a primary amenorrhea, we retained the diagnostic of FSHr-linked POI, taking in account the sudden diminution of her ovarian reserve.

With the fertility cryopreservation by oocyte vitrification, a total of 8 metaphase II oocytes ( 24 hours post IVM) and 2 metaphase I oocytes (post oocyte retrieval) were successfully vitrified and cryopreserved. A total of 3 IVM cycles were necessary for the obtention of a total of 10 oocytes.

\section{Discussion}

To our knowledge, this is the first reported successful fertility preservation by combining oocyte-IVM and vitrification for a POI patient. The main purpose of our case report is thus to suggest an opportunity for fertility preservation using 
IVM in the management of patients with confirmed POI in the frame of a FSHR mutation. Grynberg et al. already mentioned that there is a place for IVM in patients with ovarian resistance to FSH. They reported two cases of live birth after IVM in such a patient [8]. Even if their FSHR status was not specified, these successes are another argument in favor of IVM for POI patients with FSHR mutations. Our enthusiasm should probably be tempered by the fact that our patient is still far from delivery, but considering the number of cryopreserved eggs (a total of 10 eggs) and her young age, her predicted probability of having one child is over $60 \%$, based on the work of Doyle et al. [9].

Being able to obtain mature oocytes in this clinical context is not a surprise. The pathway that prevents germinal vesicle (GV) and oocyte maturation is well characterized to comprise signaling from the granulosa cells (GC) that ultimately keeps cAMP high in the oocyte until communication with the GC is lost. In vivo this loss of communication normally happens because of the LH surge. But physical removal of GV oocytes from the follicle, as in our situation, has the same effect, lowering oocyte cAMP, and thus triggering oocyte maturation. This approach is used routinely experimentally to trigger oocyte maturation in many mammalian systems. Thus, it is no surprise that, even in the context of altered FSH signaling, any oocytes that managed to make it to a threshold size were capable of resuming meiosis [10] [11] [12].

Before aiming for fertility preservation, one should first obtain the correct diagnosis of POI and, possibly, more precisely the rare condition of FSHR mutation in order to anticipate an ulterior ovarian failure in these unusual patients with amenorrhea. As for our patient, the diagnosis of POI was first suspected when a primary amenorrhea was associated with a hypergonadotropic hypogonadism, a normal AFC and a normal AMH. Such situations were formerly investigated with invasive techniques like an ovarian biopsy [13], in the certainty of a negative screening for FMR1 mutation or permutation [14] and a normal karyotype [15], trying to characterize a histological anomaly that could explain the clinical presentation. Of course, the possibility of genetic testing and the potential association of genetic mutation with POI open a new window of opportunity in the diagnosis and the support of these patients [16] [17]. In our case, no ovarian biopsies were available, thus no correlation was possible between the mutated receptor function and the ovarian histology.

Beau et al. have already and for the first time mentioned the reported missense mutation of our patient in 1998. It has to be clarified that their patient was found to carry a compound heterozygotic mutation affecting the FSHr gene and resulting in an Ile160Thr and an Arg573Cys substitutions. These substitutions were located, respectively, in the extracellular domain and in the third intracellular loop of the receptor. Their patient presented with another phenotype as she had secondary amenorrhea and normal sized ovaries [6] [18]. Also, Binder et al. [19] found that heterozygotic mutation Ile160Thr was carrying in 5.6\% - 6.1\% of infertile patients. But our patient is the first reported case of a homozygosity for the variant c.479T > C, suggesting that this condition could also lead to a POI. 
Of course, amenorrhea or even POI are not obligatorily caused by a FSHR mutation. Achrekar et al. screened the entire FSHR gene in a population of Indian women with primary amenorrhea and found none of the known mutations. They identified a particular polymorphism that was more often present in women with elevated gonadotropins [20].

More recently, Desai et al. summarized the known FSHR mutation, their effect on the receptor function and the repercussion on the reproductive system for the affected woman [4]. This work suggests that the mutations are activating or inactivating. Inactivating mutations impair either the extracellular domain of the FSHR, resulting in the diminution of its expression at the ovarian cell surface, its ability to bind the ligand being not affected, or the affinity of the receptor to the ligand [4] [21]. In both situations, the formation of the receptor-ligand complex or the FSH signals transduction pathway would be altered ending to a diminution of the FSH induced cAMP production. But, in case of a diminished affinity, a higher gonadotropin concentration could partially solve the problem, which is not the case if the expression itself is impaired. If activating, the mutation was related to OHSS or even spontaneous OHSS.

Being convinced that IVM is worth considering as fertility preservation in young patients with POI, another interesting approach suggested by Suzuki et al. deserves attention. They report ovarian tissue cryopreservation by vitrification, followed by in vitro activation (IVA) of dormant follicles in POI patients with residual ovarian follicles. After activation, pieces of ovarian tissue are grafted back in the mesosalpinx and stimulated to obtain mature follicles. Out of their initial 37 patients, 20 had residual follicles. 9 of the 20 showed a follicular growth as a response to the post graft stimulation, and 24 oocytes were collected from 6 patients. Finally, 2 term pregnancies were obtained from these oocytes [22].

\section{Conclusion}

POI patients secondary to FSHR mutation are probably underdiagnosed due to the lack of readily available testing. FSHR mutation should be considered in patients with normal-high ovarian reserve (AMH and AFC) and discrepant FSH/LH results. We suggest that genetic analysis and further diagnosis precision should be more often investigated in these situations.

New hope is arising for patients carrying the FSHR gene mutation with POI, since we and others are showing encouraging results of fertility preservation for these patients. Being the first to report a case of successful oocyte vitrification following IVM in a patient with FSHr mutation, we call for further studies to assess this original approach and improve its success.

\section{Acknowledgements}

The authors gratefully thank the OVO R \& D unit for their technical help.

P.-A. Pradervand thanks the SICPA foundation and the Société Académique Vaudoise for their support. 


\section{References}

[1] van Kasteren, Y.M., Hundscheid, R.D., Smits, A.P., Cremers, F.P., van Zonneveld, P. and Braat, D.D. (1999) Familial Idiopathic Premature Ovarian Failure: An Overrated and Underestimated Genetic Disease? Human Reproduction, 14, 2455-2459. https://doi.org/10.1093/humrep/14.10.2455

[2] Orlandini, C., Regini, C., Vellucci, F.L., Petraglia, F. and Luisi, S. (2015) Genes Involved in the Pathogenesis of Premature Ovarian Insufficiency. Minerva Ginecologica, 67, 421-430.

[3] Aittomaki, K., Lucena, J.L., Pakarinen, P., Sistonen, P., Tapanainen, J., Gromoll, J. et al. (1995) Mutation in the Follicle-Stimulating Hormone Receptor Gene Causes Hereditary Hypergonadotropic Ovarian Failure. Cell, 82, 959-968. https://doi.org/10.1016/0092-8674(95)90275-9

[4] Desai, S.S., Roy, B.S. and Mahale, S.D. (2013) Mutations and Polymorphisms in FSH Receptor: Functional Implications in Human Reproduction. Reproduction, 146, 235-248. https://doi.org/10.1530/REP-13-0351

[5] Conway, G.S., Conway, E., Walker, C., Hoppner, W., Gromoll, J. and Simoni, M. (1999) Mutation Screening and Isoform Prevalence of the Follicle Stimulating Hormone Receptor Gene in Women with Premature Ovarian Failure, Resistant Ovary Syndrome and Polycystic Ovary Syndrome. Clinical Endocrinology, 51, 97-99. https://doi.org/10.1046/j.1365-2265.1999.00745.x

[6] Beau, I., Touraine, P., Meduri, G., Gougeon, A., Desroches, A., Matuchansky, C., et al. (1998) A Novel Phenotype Related to Partial Loss of Function Mutations of the Follicle Stimulating Hormone Receptor. The Journal of Clinical Investigation, 102, 1352-1359. https://doi.org/10.1172/JCI3795

[7] Mohiyiddeen, L., Nardo, L.G. (2010) Single-Nucleotide Polymorphisms in the FSH Receptor Gene and Ovarian Performance: Future Role in IVF. Human Fertility, 13, 72-78. https://doi.org/10.3109/14647271003632322

[8] Grynberg, M., El Hachem, H., de Bantel, A., Benard, J., le Parco, S., Fanchin, R. (2013) In Vitro Maturation of Oocytes: Uncommon Indications. Fertility and Sterility, 99, 1182-1188. https://doi.org/10.1016/j.fertnstert.2013.01.090

[9] Doyle, J.O., Richter, K.S., Lim, J., Stillman, R.J., Graham, J.R. and Tucker, M.J. (2016) Successful Elective and Medically Indicated Oocyte Vitrification and Warming for Autologous in Vitro Fertilization, with Predicted Birth Probabilities for Fertility Preservation According to Number of Cryopreserved Oocytes and Age at Retrieval. Fertility and Sterility, 105, 459-466.

https://doi.org/10.1016/j.fertnstert.2015.10.026

[10] Tsafriri, A., Chun, S.Y., Zhang, R., Hsueh, A.J. and Conti, M. (1996) Oocyte Maturation Involves Compartmentalization and Opposing Changes of cAMP Levels in Follicular Somatic and Germ Cells: Studies Using Selective Phosphodiesterase Inhibitors. Developmental Biology, 178, 393-402.

https://doi.org/10.1006/dbio.1996.0226

[11] Jaffe, L.A. and Norris, R.P. (2010) Initiation of the Meiotic Prophase-to-Metaphase Transition in Mammalian Oocytes. In: Verlhac, M.-H. and Villeneuve, A., Eds., Oogenesis: The Universal Process, John Wiley \& Sons, Ltd., Chichester, 181-197. https://doi.org/10.1002/9780470687970.ch7

[12] Eppig, J.J. and Downs, S.M. (1984) Chemical Signals that Regulate Mammalian Oocyte Maturation. Biology of Reproduction, 30, 1-11.

https://doi.org/10.1095/biolreprod30.1.1 
[13] Nelson, L.M., Anasti, J.N., Kimzey, L.M., Defensor, R.A., Lipetz, K.J., White, B.J., et al. (1994) Development of Luteinized Graafian Follicles in Patients with Karyotypically Normal Spontaneous Premature Ovarian Failure. The Journal of Clinical Endocrinology and Metabolism, 79, 1470-1475.

[14] Voorhuis, M., Onland-Moret, N.C., Janse, F., Ploos van Amstel, H.K., Goverde, A.J., Lambalk, C.B., et al. (2014) The Significance of Fragile X Mental Retardation Gene 1 CGG Repeat Sizes in the Normal and Intermediate Range in Women with Primary Ovarian Insufficiency. Human Reproduction, 29, 1585-1593.

https://doi.org/10.1093/humrep/deu095

[15] Ferrarini, E., Russo, L., Fruzzetti, F., Agretti, P., De Marco, G., Dimida, A., et al. (2013) Clinical Characteristics and Genetic Analysis in Women with Premature Ovarian Insufficiency. Maturitas, 74, 61-67. https://doi.org/10.1016/j.maturitas.2012.09.017

[16] Touraine, P., Beau, I., Gougeon, A., Meduri, G., Desroches, A., Pichard, C., et al. (1999) New Natural Inactivating Mutations of the Follicle-Stimulating Hormone Receptor: Correlations between Receptor Function and Phenotype. Molecular Endocrinology, 3, 1844-1854. https://doi.org/10.1210/mend.13.11.0370

[17] Allen, L.A., Achermann, J.C., Pakarinen, P., Kotlar, T.J., Huhtaniemi, I.T., Jameson, J.L., et al. (2003) A Novel Loss of Function Mutation in Exon 10 of the FSH Receptor Gene Causing Hypergonadotrophic Hypogonadism: Clinical and Molecular Characteristics. Human Reproduction, 18, 251-256.

https://doi.org/10.1093/humrep/deg046

[18] Dufau, M.L., Tsai-Morris, C.H., Hu, Z.Z. and Buczko, E. (1995) Structure and Regulation of the Luteinizing Hormone Receptor Gene. The Journal of Steroid Biochemistry and Molecular Biology, 53, 283-291. https://doi.org/10.1016/0960-0760(95)00115-G

[19] Binder, H., Strick, R., Zaherdoust, O., Dittrich, R., Hamori, M., Beckmann, M.W., et al. (2012) Assessment of FSHR Variants and Antimullerian Hormone in Infertility Patients with a Reduced Ovarian Response to Gonadotropin Stimulation. Fertility and Sterility, 97, 1169-1175. https://doi.org/10.1016/j.fertnstert.2012.02.012

[20] Achrekar, S.K., Modi, D.N., Meherji, P.K., Patel, Z.M. and Mahale, S.D. (2010) Follicle Stimulating Hormone Receptor Gene Variants in Women with Primary and Secondary Amenorrhea. Journal of Assisted Reproduction and Genetics, 27, 317-326. https://doi.org/10.1007/s10815-010-9404-9

[21] Gromoll, J., Simoni, M., Nordhoff, V., Behre, H.M., De Geyter, C. and Nieschlag, E. (1996) Functional and Clinical Consequences of Mutations in the FSH Receptor. Molecular and Cellular Endocrinology, 125, 177-182. https://doi.org/10.1016/S0303-7207(96)03949-4

[22] Suzuki, N., Yoshioka, N., Takae, S., Sugishita, Y., Tamura, M., Hashimoto, S., et al. (2015) Successful Fertility Preservation Following Ovarian Tissue Vitrification in Patients with Primary Ovarian Insufficiency. Human Reproduction, 30, 608-615. https://doi.org/10.1093/humrep/deu353 